Transversal: International Journal for the Historiography of Science 2019 (7): 58-69

ISSN 2526-2270

www.historiographyofscience.org

Belo Horizonte - MG / Brazil

(c) The Author 2019 - This is an open access article

\title{
Article
}

\section{Before Foucault: The Proofs of the Earth's Rotation}

Roberto Mantovani ${ }^{1}$

\begin{abstract}
:
We are going to trace the ideas and experiments, since Galileo and until Léon Foucault, aimed at proving the Earth's rotation. Galileo - incorrectly - tried to explain the phenomenon of tidal forward and backward flow with the Earth's rotation. After Galileo, the cannon shots towards the zenith and the experiments on falling objects were considered reliable evidence. At the end of the XVII century, Newton had the idea of showing the Royal Society, through Hooke, the proof of the eastward deflection of a falling body from a considerable height. In the XVIII century, new geophysical proofs of the Earth's rotation were available. At the end of the century, in Italy, Guglielmini measured the deviations towards the east and south of small leaden balls falling inside the Asinelli Tower. The experiment was first repeated in other Italian cities and then, in the first decades of the XIX century, it was proposed again in Germany. Theoretically Laplace and Gauss, though with different approaches, came to the correct mathematical interpretation of the phenomenon. In the following years new mechanical proofs were sought but the effects due to earth rotation were too small to provide any certainty. Only Foucault managed to solve the problem.
\end{abstract}

Keywords: Historiography of Earth's rotation, experimental proofs Earth's rotation, falling bodies, Guglielmini, Benzenberg, Reich

Received: 18 June 2019. Reviewed: 25 November 2019. Accepted: 30 November 2019.

DOI: http://dx.doi.org/10.24117/2526-2270.2019.i7.05

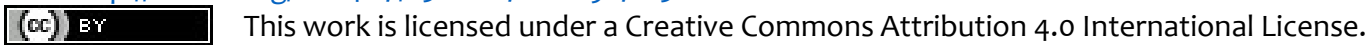

\section{Introduction}

Non, il n'y a pas d'espace absolu; ces deux propositions contradictoires: "la Terre tourne" et "la Terre ne tourne pas" ne sont donc pas cinématiquement plus vraies l'une que l'autre. Affirmer l'une en niant l'autre, au sens cinématique, ce serait admettre l'existence de l'espace absolu. Mais si l'une nous révèle des rapports vrais que l'autre nous dissimule, on pourra néanmoins la regarder comme physiquement plus vraie que l'autre, puisqu'elle a un contenu plus riche. Or à cet égard aucun doute n'est possible. Voilà le mouvement diurne apparent des étoiles, et le mouvement diurne des autres

\footnotetext{
${ }^{1}$ Roberto Mantovani [Orcid:0000-0003-3644-6605] is an Assistant Professor in the Department of Pure and Applied Sciences (DiSPeA), Physics Laboratory: Urbino Museum of Science and Technology, University of Urbino Carlo Bo. Address: Collegio Raffaello, Piazza della Repubblica 13, 61029 Urbino (PU), Italy. E-mail: roberto.mantovani@uniurb.it
} 
corps célestes, et d'autre part l'aplatissement de la Terre, la rotation du pendule de Foucault, la giration des cyclones, les vents alizés, que sais-je encore? Pour le Ptoléméien, tous ces phénomènes n'ont entre eux aucun lien; pour le Copernicien, ils sont engendrés par une même cause. En disant, la Terre tourne, j'affirme que tous ces phénomènes ont un rapport intime, et cela est vrai, et cela reste vrai bien qu'il n'y ait pas et qu'il ne puisse y avoir d'espace absolu. (Poincaré 1905, 297-298)²

As well stated by Poincaré in this passage, the Copernican theory proved to be superior to the Ptolemaic one because it was able to provide "rapports ... physiquement plus vraie" (Reports ... physically more real). In particular, the recognition of a common causal link among the several phenomena concerning the question of the Earth's rotation was at the bottom of its progressive historical success, even if its path was neither easy nor short. Also, the concepts of motion and "absolute space" gradually underwent a reassessment. Aristarchus of Samos's heliocentric theory (310-230 a. C.) introduced the relativity of motion in relation to fixed stars. Archimedes, in Arenarius, relied on the Earth's motion of Aristarchus's theory to deduce the diameter of the sphere of fixed stars from the absence of stellar parallaxes. According to this logic, he considered the Earth's motion not "absolute" with respect to an empty space but referred to the privileged system of the fixed stars. During the Hellenistic age, other ideas used the relativity of motion. Overlooking Heraclides Ponticus, Euclid, in proposition 51 of his Optics, had already observed that some "appearances" depended on the relative motion between the observer and the observed object. Lucretius, in De Rerum Natura, had described the relative motion of a ship with reference to the mainland. However, the beginning of the Aristotelian-type Ptolemaic astronomy and its theory of stationary earth marked a clear breach with the past. One of its most dramatic consequences was the loss of the idea of the relativity of motion and consequently the loss of the heuristic function that most of all the concept of "system of reference" was slowly acquiring. We will have to wait for Galileo's relativity of motion for this topic to resurface again.

\section{Galileo's Considerations}

It was during Galileo's time that the debate on the Earth's rotation took shape, most of all in relation to the verification and confutation of the Copernican system. Galileo was among the first to indicate the indirect cause of the Earth's rotation in a natural phenomenon. As a matter of fact, Galileo, though incorrectly, tried to explain the phenomenon of the tidal forward and backward flow through the Earth's rotation and its revolution around the sun. This idea, already in a letter ${ }^{3}$ dated 8 January 1616 , sent by Galileo to Cardinal Orsino, will later be reconsidered and defined by Galileo as irrefutable in the Dialogue Concerning the Two Chief World Systems of 1632 (day four). Several times in this work (see especially day two), Galileo

\footnotetext{
${ }^{2}$ No, there is no absolute space; these two contradictory propositions: "the Earth turns round" and "the Earth does not turn round" are, therefore, neither of them more true than the other. To affirm one while denying the other, in the kinematic sense, would be to admit the existence of absolute space. But if the one reveals true relations that the other hides from us, we can nevertheless regard it as physically more true than the other, since it has a richer content. Now in this regard, no doubt is possible. Behold the apparent diurnal motion of the stars, and the diurnal motion of the other heavenly bodies, and besides, the flattening of the Earth, the rotation of the Foucault's pendulum, the gyration of cyclones, the trade-winds, what not else? For the Ptolemaist all these phenomena have no bond between them; for the Copernican they are produced by the one same cause. In saying, the Earth turns round, I affirm that all these phenomena have an intimate relation, and that is true, and that remains true, although there is not and can not be absolute space. (Poincaré 1907 [1905], 141)

3 This letter was entitled "Discourse on the flow and reflow of the sea". See (Galilei, 1895).
} 
also deals with the falling objects in relation to the controversy on the Earth's rotation. The Peripatetics' theory was that if the earth rotated then the falling bodies, without initial velocity, would deviate towards the west from the vertical. But that could not be observed. Experience showed that a stone falling from a tower moved "perpendicularly and not obliquely" as in the case of a constantly moving earth. Galileo skilfully demolishes this empirical thesis, adds transverse motion - due to the Earth's rotation - to the vertical one without, however, managing to describe a parabolic motion. He also explains that we do not perceive transverse velocity simply because we move in the same system of reference (the famous thought experiment of the "large ship"). He, therefore, proves that the falling objects cannot be considered as a proof against a moving Earth. Much vaguer was, on the contrary, his standing on the measure of a deviation of the body towards the east, which Galileo thinks it is possible to observe, but only for great distances. In this regard, he describes an ideal experiment (a ball falling from the Moon to Earth) neither providing a rigorous illustration nor suggesting a probative experiment. (Galileo 1632, second day, Salviati, 220-221, 228-229).

\section{Other Contributions from the XVII Century}

In the first half of the XVII century, other scientists tried different ways to demonstrate the Earth's rotation. In a letter of 1643 Pierre Gassendi (1592-1655) reports the daily attempts made by Alexandre Calignon de Peyrins (1589-1656) to detect small deviations from the vertical of some perpendicular threads of different lengths. Also ineffective were the attempts of shots towards the zenith with artillery cannons meant to show the westbound movement of the cannon balls when falling to the ground. The most famous were the ones made, following Descartes's suggestions, by Father Marin Mersenne (1588-1648) and by the ingénieur du Roi et intendant des fortifications Pierre Petit (1598-1667), in the surroundings of Paris in the late spring of 1638. In the second half of the XVII century, worthy of remark were Viviani's (1661) experiments on the motion of pendulums in Florence, the Italian controversy on the trajectory of a falling object in "absolute space" (Gregory 1668, 693-698) whose protagonists were on one hand the Galilean Giovanni Alfonso Borrelli (1608-1679) and Stefano degli Angeli (1623-1697) and, on the other, the Jesuit Giambattista Riccioli (1598-1671), and, most of all, the exchange of letters (1679-1680) between Isaac Newton and Robert Hooke on the falling bodies.

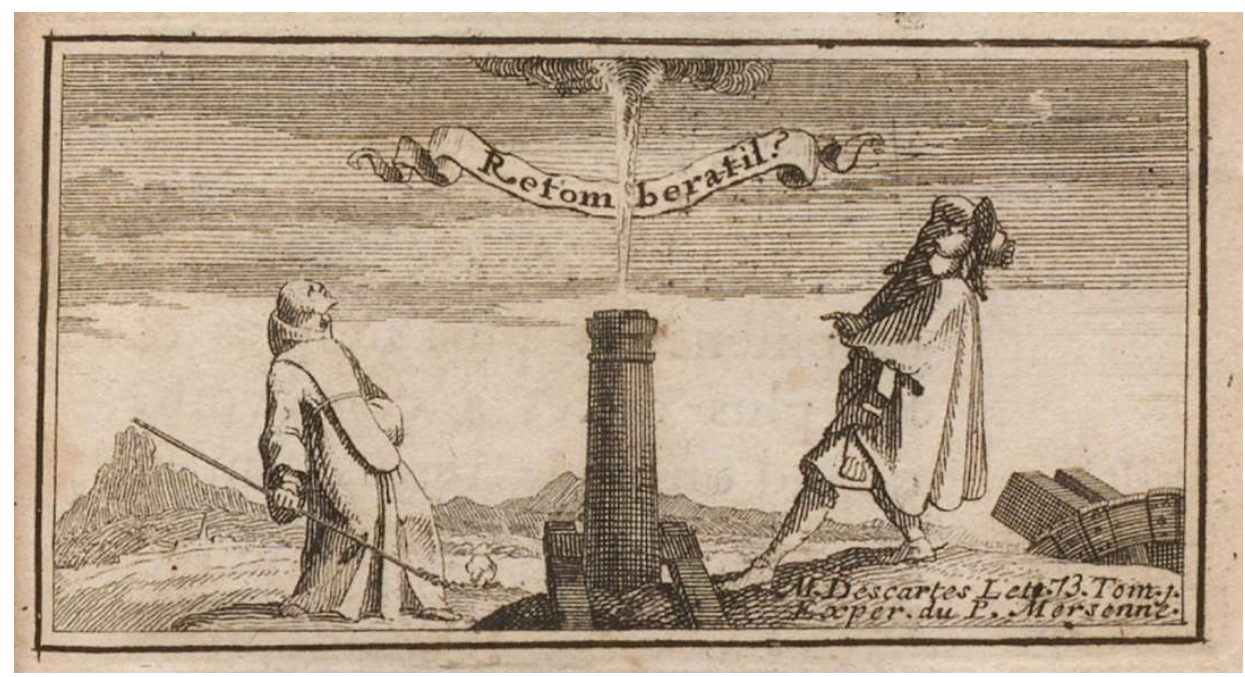

Fig. 1 Mersenne (left) and Petit use a vertical cannon to prove the earth rotation (Varignon 1690) 


\section{Viviani's Experiment}

The Academics of Experiment produced experiments on the Earth's rotation but did not publish them and probably did not even fully understand their meaning and scientific importance. Substantial evidence can be found in a handwritten note by the secretary of the Academy, Vincenzo Viviani (1622-1703), dated 1661, where there is the description of the plane rotation of some pendulums together with an explicative picture showing the counterclockwise rotation of the plane. Next to the picture, there were these words: "We observed that all the pendulums from one thread deviate from the first vertical, and always in the same direction, i.e. according to the lines $A B, C D$, EF, etc. from right to left of the back parts, etc." This note was discovered by Vincenzo Antinori in Florence in 1851, soon after the famous experiment of Foucault's pendulum in Paris. Antinori (1792-1865), who was at the time Director of the Imperial Royal Museum of Physics and Natural History of Florence, announced the discovery in a letter addressed to the astronomer François Arago (1786-1853), continuous secretary of the Académie des Sciences of Paris. The same letter was then published in the Comptes Rendus de l'Académie des Sciences (Antinori 1851b, 635-636), inside the instalment of the announcement of the official discovery of Foucault's pendulum rotation. The news of Antinori's claim was delivered with great prominence in Italy, feeding a patriotic feeling among pre-unification scientists and a sense of vengeance by the Italian science towards foreign discoveries.

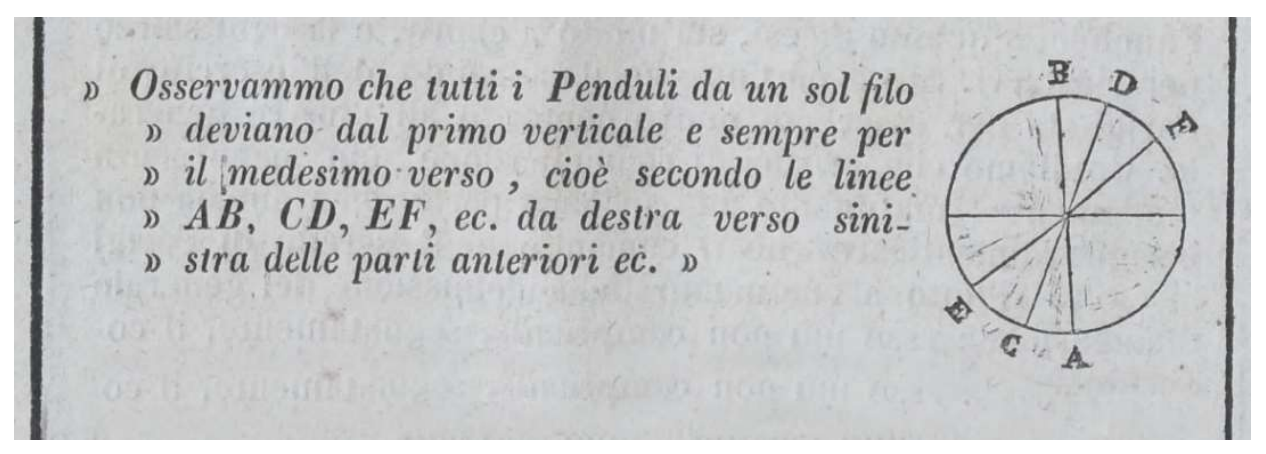

Fig. 2 Unpublished note written by Viviani and reported for the first time by Antinori in the Florentine newspaper "Lo Statuto" (Antinori 1851a, 2)

\section{Newton, Hooke and the Deviation towards the east of Falling Bodies}

The anti-Copernican Riccioli, in his monumental astronomical work Almagestum Novum of 1651, had already produced a great number of experiments on falling bodies (Graney, 2012). One of these experiments, ${ }^{4}$ taken as a proof of the immobility of the Earth and devised to discredit an opinion of Galilei's, was the object of much debate with Angeli and Borrelli. Most of all, it had the merit of highlighting the deviation towards the east of freely falling bodies from the vertical of the shooting point (Borgato, 2011). This debate was accurately reported and published in the Philosophical Transactions of 1668 by the Scottish mathematician and astronomer James Gregory (1638-1675), a member of the Royal Society. It may have been thanks to this report that Newton had the idea of proposing the Royal Society, through Hooke, a check of the deviation towards the east of the freely falling bodies from a great height. As already mentioned, before Newton this experiment, because of its poor results, had been discussed and used by the anti-Copernicans more to question the Earth's rotation

\footnotetext{
4 The experiment specifically dealt with the intensity variation of the impact to the ground of a falling body according to its height fall.
} 
than to highlight it. For the first time, Newton reverses this trend and presents it as a direct mechanical evidence of the Earth's rotation. The occasion for that was an answer Newton gave the Secretary of the Royal Society, Robert Hooke (1635-1703), to some questions about celestial mechanics. In that letter, dated November 1679, Newton expressed the idea of checking the daily Earth's rotation with a precise experimental test (Ball 1893, 141-144). He maintained that if you let a body fall from a great height, it will fall to the east from the vertical at the starting point. According to Newton, this movement derived from the fact that, at the moment it fell from a given height, the object had a tangential velocity given by the Earth's rotation greater than the one it had when it reached the foot of the vertical. With a simple calculation, the lateral movement was obtained by multiplying the fall time by the difference between the two velocities. This first simplified model provided a move towards the east which was overestimated because it overlooked the curvature of the Earth and other important parameters. But it had the advantage of highlighting the correct prediction that the falling object would have touched the ground in a point further east from the vertical. Oddly, Newton added "a fancy of my own" - an ideal experiment - to this real experiment. He imagined that, if the body could have kept on running beyond its ground zero, that is the interior of the Earth, it would have reached the core of the Earth along a spiral trajectory. Newton's letter was read and discussed on December 4, 1679, during a meeting at the Royal Society, where the experiment of the falling objects was very well received. On December 9, 1679, Hooke wrote an answer to both of Newton's questions. According to his calculations, the object would not fall to the centre of the Earth; moreover, the trajectory was more like an ellipse than a spiral. ${ }^{5}$ In relation to the question of the freely falling body, Hooke observed that the fall "will not be exactly east of the perpendicular but South East and indeed more to the south than the east" (Turnbull 1960, Vol. II, 306). To support his idea in the following days he realised some outdoor trials and, in a letter dated January 6, 1680, he said to Newton: "I must acquaint you that I have (with as much care as I could) made 3 trials of the experiment of the falling body, in every of which the ball fell towards the south-east of the perpendicular, and that very considerably, the least being above a quarter of an inch [about $0,65 \mathrm{~mm}$ ], but because they were not all the same I know not which was true" (Ball 1893, 148). On January 16 , to attenuate the effects of the interference of air currents, Hooke made two new indoor trials whose results were, according to him, satisfying (Robinson, Adams 1935, 435). Indeed, Hooke wrote to Newton, in a letter of January 17, 1680: "I am now persuaded the experiment is very certain, and that it will prove a demonstration of the diurnal motion of the earth as you have very happily intimated" (Ball 1893,149 ) and some days later, Hooke ${ }^{6}$ reaffirmed in his diary: "Diurnal motion of Earth established" (Robinson, Adams 1935, 436). It is interesting to underline that Hooke, even though he did not provide meaningful experimental data, was the first who introduced the idea of the southern deviation in the experiment of freely falling bodies.? The authority of Newton and Hooke produced a solid reputation for the experiment of the falling objects, which was repeated several times until Foucault's days and was considered one of the main direct mechanical evidence of the Earth's rotation.

\footnotetext{
${ }^{5}$ This well-known debate basically was about the famous inverse-square law; few years later, it brought to the publishing of "Principia" but also to Hooke accusing Newton of plagiarism. See (Westfall 1989, 399-404).

${ }^{6}$ To be thorough, we must remember that, in 1764, Hooke had published "An attempt to prove the motion of the earth from observations" where, besides presenting his personal "System of the World", he had tried to demonstrate the motion of the Earth in the space through measures of stellar parallaxes.

7 This move derives essentially from that part of the centrifugal force of the Earth's rotation oriented according to the local meridian, towards the geographical south. This part, calculated by Gauss and Laplace at the beginning of the XIX century, became the greater as the more pronounced the fall height was and smaller the latitude of the place of the experiment (see note n. 15).
} 


\section{The Geophysical Proofs from the XVIII Century}

After Hooke's experiment, we will have to wait for the end of the XVIII century for another meaningful experimental test like that in Italy. Meanwhile, in the XVIII century, new indirect geophysical evidence of the Earth's rotation became available. Among these, we remember how gravitational acceleration decreases from the poles to the equator and the study of the shape of the Earth. The scientific journeys had the merit of highlighting the variation of gravity due to latitude. By getting information from one of these travels, ${ }^{8}$ Newton pointed out that pendulum oscillations were slower close to the equator than those at the poles and, in the first edition of "Principia" (1687), he decided that the reason why the Earth is flattened at the poles ${ }^{9}$ is due to the centrifugal force of the Earth's rotation whose strength decreased as latitude increased. This conclusion created controversy in the Continent. The "querelle" on the shape of the Earth burst in France between Cartesians and Newtonians. In 1732 PierreLouis Moreau de Maupertuis (1698-1759) supported Newton's opinion that the Earth, because of its own motion, should have been a spheroid flattened at its poles (Fig.3). Two famous geodesic missions, organized by the Académie Royale des Sciences, one near the equator in Peru (1735), the other near the arctic circle, in Lapland (1736), largely proved Newton's theory - supported by Maupertius - right: the arc of the meridian measured in Lapland was longer than the one in Peru. Therefore, thanks to the introduction of new measuring methods, notably the dynamic method (pendulum oscillations) and the geometric method (measuring the arcs of a meridian), the scientific community was able to produce two important experimental proofs of the Earth's rotation. Anyway, they were still indirect proofs.

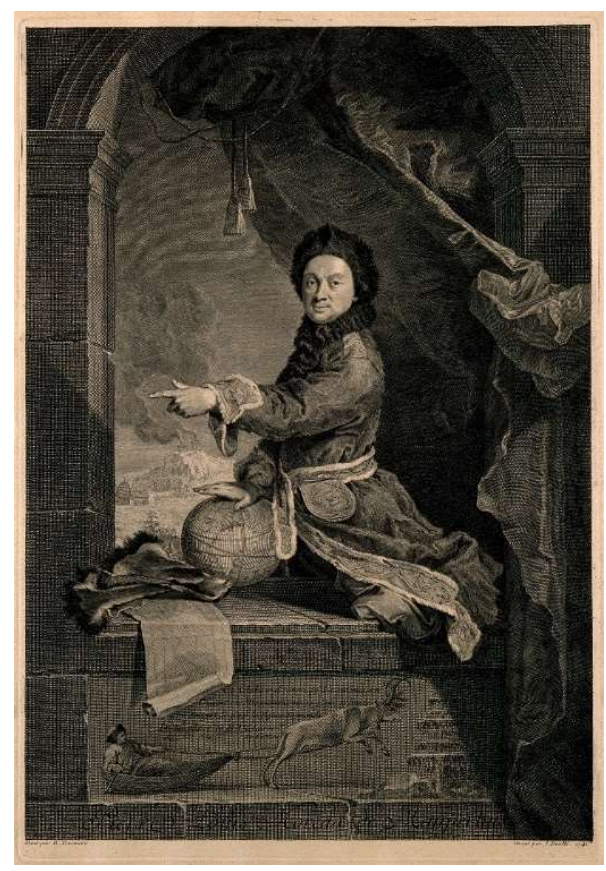

Fig. 3 Maupertuis shows the crushing of the earth with his left hand (engraving by J. Daul Wellcome)

\footnotetext{
${ }^{8}$ It is the French scientist Jan Richer's journey to the island of Cayenne, near the equator. During his stay on the island, in the years $1672-1673$, he observed that his pendulum clock, previously set in Paris, went slower.

${ }^{9}$ Newton came to this conclusion also thanks to some astronomical observations by John Flamsteed and Jean-Dominique Cassini on the flattening of Jupiter at its poles (Greenberg 1987, 357).
} 


\section{The Falling Objects in Italy 1790-1795}

At the end of the XVIII century, the "direct" experiment proposed by Newton in 1679 was reconsidered. A group of Italian mathematicians and astronomers devised and repeated the experiment of the deviation of freely falling bodies from great height in several Italian cities. It originated an intense scientific debate that also produced the first simplified theoretical models of the phenomenon. The experiment which triggered the debate and the following other experiments was realized by the physicist and religious Giambattista Guglielmini (1760-1817) from Bologna; at first, it was designed for St. Peter's Basilica in Rome (1789) and then actually re-designed and implemented between 1790 and 1792 in Bologna, at the Asinelli Tower. Guglielmini measured the eastward and southward deflections of small lead balls freely falling inside the tower for 78.3 metres. The results of the experiment, from the vertical identified by the plumb line, provided an easterly mean deviation of $18.894 \mathrm{~mm}$ and a southerly mean deviation of $11,894 \mathrm{~mm}$ (Guglielmini 1792, 82). New accurate experiments followed in other Italian cities: in Rome (Calandrelli, tower of the Observatory of the Roman College, 1790-1791); in Novara (Teresio Michelotti, Bell tower of Saint Gaudenzio); in Turin (Félix de Saint Martin de la Motte, Basilica of Superga, 1791) and finally in Bergamo (Tadini, Basilica of Santa Maria Maggiore and bell tower of the Franciscan Convent, 1794-1795). During this short but intense period of experiments, hypotheses and theoretical models proceeded on successive approximations, thanks also to new mathematical and experimental techniques. Active participants in the debate were Sebastiano Canterzani (1734-1818) from Bologna, mathematician and teacher of Guglielmini, Teodoro Bonati (1724-1820), professor of Mechanics and Hydraulics at the University of Ferrara, the brothers Teresio (1762-1819) and Ignazio (1764-1846) Michelotti from Turin, both hydraulic and mechanical engineers, Girolamo Saladini (1735-1813), mathematician and professor of Calculus at the University of Bologna, Giuseppe Calandrelli (1749-1827), astronomer at the Observatory of the Roman College in Rome and Abbot Gianantonio Tadini (1754-1830), professor of Physics at the Marian College of Bergamo. With this experiment, we can state that, as a whole, towards the end of the XVIII century, in Italy, a good number of theoretical studies, supported by intense experimental activity, weaved together in a virtuous circle. The theoretical analyses, at first rather incomplete and incorrect, more and more considered several parameters of the experiment, such as the spherical and spheroidal shape of the Earth, the eastward and southward deviations (the latter, in particular, thanks to Saladini's works), the uniform and central gravitational field, the resistance of the medium both for vertical falling and the eastward, the theoretical model of fall through a vacuum, the deviation of plumb line, the centrifugal force. To remove the initial vibrations they improved the mechanical device dropping the falling objects, and the mathematical methods were perfected, too: Tadini, for instance, instead of geometrical analysis, used differential calculus for his calculations and, though with some theoretical mistakes, reached "the same identical result of Laplace, probably also thanks to an odd compensation of mistakes"10 (Borgato 2007, 521).

\section{Laplace, Gauss and the Mathematization of Falling Objects}

In the last years of the XVIII century, theoretical ideas and the results of the experiments carried out in Italy were resumed and discussed in Europe, most of all in France and

\footnotetext{
${ }^{10}$ Tadini had come to the conclusion that, despite the action of a small component of the resistance of the air orthogonal to the plumb line, the southward deflection would have been null both in a vacuum and in the air. This conclusion will be validated by Laplace in 1803. To be noted that Guglielmini had instead considered the southward deviation null in a vacuum but not in the air because of the determining role of the resistance of the air, according to him (Giannini 2015, 326).
} 
Germany, thanks to the mediations of the French astronomer Jérôme Lalande (1732-1807) and of the German physicist and mathematician Benzenberg (1777-1846), professor of Astronomy and Physics in Düsseldorf. In France, the debate generated important theoretical results, thanks to Pierre Simon Laplace (1749-1827). By applying the analytical method, since 1796, he had already formulated the correct mathematical theory of the falling objects. However, his results were published later on: first in a basic paper of 1803 (Laplace 1803) where he exclusively dealt with the vertical fall of objects, and then, with no substantial changes, in a chapter of his famous work Traité de Mécanique Céleste (Laplace 1805), where he dealt with the more general problem of projectile motion ${ }^{11}$. In the same years, in Germany, Benzemberg, encouraged by the news form Lichtenberg about Guglielmini's experiment, repeated the same experiment first in 1802 in Hamburg, inside the bell tower of St. Michael Church, and then two years later in a shaft of an abandoned coal mine ${ }^{12}$ at Schlebusch, near Leverkusen in Germany. In Hamburg, with an available height of 76.3 metres, Benzemberg found average deflections towards the east and the south respectively of $9 \mathrm{~mm}$ and $3.4 \mathrm{~mm}$; at Schlebusch, with an available 84.4-metre-deep mineshaft, the average eastward deflection was $8.5 \mathrm{~mm}$, while the southward was null (Cajori, 1901, 853). Benzemberg transmitted the experimental data of the first experiment to the astronomer Wilhelm Olbers (1758-1840), who showed them to Carl Friedrich Gauss (1777-1855) who became involved in this way in the theoretical study of falling objects. So, almost at the same time, a young Gauss and a more mature Laplace happened to work on the same topic. ${ }^{13}$ Though with different approaches, they both came, with slight differences, to the correct mathematical interpretation of the phenomenon. Their theoretical contributions, before Foucault's experience, were fundamental to formulate the general theory of falling objects and to make Newton's old experience more convincing. These two mathematical approaches deserve some more thought. Laplace and Gauss worked at elaborating three differential equations of motion and used some approximations. ${ }^{14}$ These equations, conveniently integrated and developed in a series, provided the equations of motion respectively of the vertical fall, the eastward deflection and the southward one. In his calculations, Laplace used the geographical colatitude; Gauss, on the other hand, relied on the geographical latitude. The two scientists studied the motion of falling objects with respect to two reference systems: the first fixed in (absolute) space and the second (relative) moving, that is integral with the Earth. The use of this second system allowed them both to come across two apparent forces, one of which, unknown at the time, proved to be responsible for the eastward deflection of falling objects. It was the well-known Coriolis force, ${ }^{15}$ dependent on the angular velocity $\omega$ of the rotating frame and on the falling object's velocity, whose official discovery is generally dated back to 1832-1835, that is more than thirty years later, in relation to the French mathematician, physicist and engineer Coriolis's technical-practical studies (the rotating machines). Ignoring the resistance of the air, the theoretical analyses of the two great

\footnotetext{
${ }^{11}$ Reading Laplace's work today is not easy. He would use Cartesian coordinates but neither vectors nor explanatory images.

${ }^{12}$ With this experiment, Benzemberg was the first to use the depth of a mine instead of the height of towers or bell towers. The aim was to reduce the experimental disturbances from air currents.

${ }^{13}$ Benzemberg sent his own experimental data to Laplace, too. It was not the first time that Laplace and Gauss matched against each other. In January 1801 the Italian astronomer Piazzi discovered a new planetoid Ceres Ferdinandea but could not calculate its orbit. With the few available astronomical data, Gauss managed to calculate its orbit whereas Laplace declared it was not possible.

${ }^{14}$ For instance: the gravity acceleration considered constant and independent from the height; the resistance of the air proportional to the square of the linear fall velocity.

15 The mathematical term related to this force appears first in a work by Laplace (Laplace, 1803) and, a year later, in a publication by Benzemberg where, in a chapter, Gauss's calculation is reported (Gauss 1804, Gauss Werke 1867).
} 
mathematicians provided essentially the same result as regards the eastward deflection of falling objects, ${ }^{16}$ that is a movement in the amount of two-thirds of Guglielmini's theoretical value. Instead, as regards the southward deflection, in vacuum, while developing the formula series, Laplace ignored the quadratic terms of the angular velocity and concluded that the southward deflection had to be considered null (Laplace 1803, 113). Gauss, conversely, chose to approximate the formula at the quadratic term of the angular velocity and found a deflection towards the equator extremely small, but finite. ${ }^{17}$ Therefore, Benzenberg's experiments gave results in accordance with the theory of Laplace and Gauss only for the eastward deflection. The data of the southward deflection were instead contradictory, and still today this kind of deflection raises more than a doubt. ${ }^{18}$ Even more accurate experiments in the deflection of falling bodies were carried out, in 1831, by the German chemist and physicist Ferdinand Reich (1799-1882) in a mineshaft in Freiberg, in Saxony, taking advantage of a remarkable depth, 158.5 metres, and a fall almost twice the one used by Benzemberg. Reich used some very resistant metal or metal-alloy balls ${ }^{19}$ with a diameter of $4 \mathrm{~cm}$. To remove humidity and currents of air, which disturbed the fall motion of the balls, Reich built a long wooden tube with a rectangular section. After six series of experiments and a good 106 tests of free fall, Reich managed to have an average southward deflection of $4.374 \mathrm{~mm}$ and, most of all, an average eastward deflection of the balls of about $28.396 \mathrm{~mm}$, a value which was very close to the theoretically calculated value of $27.5 \mathrm{~mm}$. These experiences were accurately described by the German scientist the following year (Reich, 1832). In their own kind, they were the last and more accurate experiences carried out in the XIX century. ${ }^{20}$

\section{From Ballistics to Foucault's Pendulum}

Despite the progressive improvements of measurements and experimental conditions ${ }^{21}$ the

${ }^{16}$ More precisely, the Easterly deviation was $d=\frac{1}{3} \omega g t^{3} \cos \varphi=\frac{2}{3} \omega h \cos \varphi \sqrt{\frac{2 h}{g}}$ where $\omega$ is the Earth's angular velocity $\left(7,292 \cdot 10^{-5} \mathrm{rad} / \mathrm{s}\right.$, i.e., $2 \pi$ divided by the sidereal day: $\left.23 \mathrm{~h} 56 \mathrm{~m} 4 \mathrm{~s}\right) ; \varphi$ is the geographical latitude; $g$ is the acceleration of gravity and $h$ is the height from which the object falls. Two years later, in his Traité de Mécanique Céleste (Laplace, 1805) Laplace dealt with the same topic considering the air resistance part.

17 The Southerly deviation value found by Gauss was $d=\frac{1}{6} \mathrm{~g} \omega^{2} t^{4} \sin \varphi \cos \varphi$ (Gauss, 1804, 370).

${ }^{18}$ The value of the southward deviation, from Guglielmini on, has always recorded measures in conflict with the theory. In 1902, the physicist Hall studied this deviation at the Harvard Physics laboratory: he dropped small bronze spheres from a height of 23 metres. The measure gave a result 500 times the Gauss result. Further experimental tests and several sophisticated theoretical explicative models were developed, at the beginning of the XX century, by Johann Georg Hagen (1912), Magnus le Comte De Sparre (1905), Maurice Fouché (1905), William H. Roever (1911-1912), R. S. Woodward (1913), Alfred Denizot (1913) and Lorand Roland Eötvös (1906-1909). As underlined by Tiersten and Soodak "Now, almost a century later, the situation remains unresolved" (Tiersten, Soodak, 2000, 130).

19 The materials were lead, tin and ivory; the alloys, on the other hand, were made by tin, bismuth and lead. When these spheres dropped, if they did not have the perfect coincidence between the barycentre and the sphere's geometric centre, they could rotate and generate a rolling friction with the air. This effect, that could distort the deviation, apparently was not considered by Reich.

${ }^{20}$ After Reich, many other experiments were performed, of little importance, focussed mainly on the study of falling bodies towards the south. Among these experiences we must remember the one carried out in 1848 by William Westcott Rundell (1792-1874), Secretary of the Royal Cornwall Polytechnic Institution, in a mine in Cornwall 400 metre deep.

${ }^{21}$ For instance, Reich used some expedients for the initial dropping of the spheres. One of these used the principle of s' Gravesande Ring. The spheres were heated in hot water, then dried and put on a circular copper ring with a slight conic shape inside. When the spheres had cooled down, they contracted and freely dropped along the inside of the wooden tube. This method, as ingenious as it 
experiments on falling objects did not provide definite evidence of the Earth's rotation. Several experimental criticalities complicated the measurements. Among these, the lack of a sure control of the currents of air during the fall, the precise determination of the vertical from great heights, the irregularities of form and density of the bodies, the vibratory motion of the bodies at the beginning of the fall. Furthermore, the measured values were too small and generated uncertainties, especially at the landing points, whose spatial distributions often showed too large a dispersion to be meaningful. Lastly, the lack of a good theory of propagation of error did not permit the statistical elaboration of the data deriving from those measurements. These problems made the experiment of falling objects inconsistent with theoretical expectations. Meanwhile, other indirect evidence of the Earth's rotation came up. The improvements of the artillery long-range gunshots imposed the mathematical study of the trajectory of a projectile in relation not only to the resistance of the air but also to the motion of the Earth's rotation. The problem was dealt with between 1837 and 1838 by the French mathematician Siméon-Denis Poisson (1781-1840). The results of these studies showed that, because of the Earth's rotation, the projectiles underwent a perceivable deviation towards the right in the northern hemisphere and towards the left in the southern. Oddly, Poisson's study on the motion of projectiles contained also a short mathematical note aimed at connecting the Earth's rotation to the kinematic motion of a pendulum. The time had come to undertake new experimental ways with the purpose of proving the Earth's rotation. Many experimenters' attention fell on the pendulum, first in its static form, the plumb line,$^{22}$ then in its dynamic version, the pendular motion. Poisson's mathematical note did not elude the physicist Jean Bernard Léon Foucault (1819-1868) who may have used it to design his famous experiment which highlighted the rotation of the plane of a swinging pendulum. The experiment was resoundingly carried out at the Panthéon in Paris on 31 March 1851 and then successfully repeated in many other French and European cities. Scientists welcomed this experiment and considered it as a new, direct and convincing evidence of the rotation of the Earth. Suddenly and rapidly all the past experiments fell into oblivion.

\section{Final Conclusion}

The historical events related to the proofs of the Earth's rotation were important right from the start, both in the events leading up to the affirmation of the Copernican system, and in the development of some concepts typical of the kinematic and dynamic theories of motion. These proofs had a strong impact in the progressive perfecting of important topics in the study of Mechanics, such as the relative motion and the use of reference systems. The phenomenon of the eastward deflection of a falling body was considered, up to Foucault's days, the most important experience validating the Earth's rotation. Anyway, this procedure eventually turned out to be essentially heuristic. The experiments carried out in Italy at the end of the XVIII century stimulated, in France and Germany, important theoretical considerations that eventually led to the correct calculation of the eastward and southward deflection of freely falling bodies. On the experimental side, the phenomenon showed several complications that made the final results very uncertain. One of the major discrepancies between theory and experiment concerned the southward deflection which, because of its extremely small value and the many external disturbing effects, could not actually be observed. All of this produced wide debate and little certainty about the proof of

was, was not free of systematic errors and other critical observations of the experimental kind, for example, the presence of small lateral impulses at the moment of the fall.

${ }^{22}$ The idea was to measure the movement of a plumb line due to the non-inertial effect of the Earth's rotation. One of the most interesting experiments was the one designed by the French physicist Guyot in 1836 at the Paris Pantheon. Guyot used a 57-metre-long plumb line and, with an optical method, verified that the line moved $4.33 \mathrm{~mm}$ from its vertical. 
the Earth's rotation. Some new mechanical evidences were then researched. The shift from a state of rest of very long plumb lines and the perceivable deviation from their trajectory of artillery long-range gunshots gave the illusion of highlighting the non-inertial effects due to the Earth's rotation. But, once again, these effects were too small to give some certainty. It was necessary to identify a pendular system which, manually activated, might have the characteristic of keeping its oscillating motion for some time or in a persistent way. This dynamic condition would have allowed the system to accumulate, in time, and show the effects of the apparent forces due to the Earth's rotation. Foucault was actually the first to identify, in the oscillations of a long string pendulum, the presence of an additional precession motion of the oscillation plane. It is worth noticing that nowadays modern physics textbooks explain this precession in terms of Coriolis force: they implicitly suggest a historical connection between this force and the 1851 experiment, but historical facts do not seem to confirm such a connection. The principles of the so-called "force centrifuge composée", enunciated between 1832 and 1835 by Gaspard-Gustave de Coriolis (1792-1843), did not inspire the pendulum experiment at all, perhaps because Foucault was not aware of them. After all, he was no mathematician and always preferred an intense technical and experimental activity to theoretical principles. His undeniable skills in "observing the phenomenon" pushed him to design an experiment which had its most effective force of persuasion in the evidence of observation.

\section{References}

Antinori, Vincenzo. 1851a. Moto del pendolo applicato alla rotazione della terra. Lo Statuto, III, 68, Mercoledì 23 Aprile: 2.

Antinori, Vincenzo. 1851b. Anciennes observations faites par les Members de l'Académie del Cimento sur la marche du pendula. Lettre de M Antinori, directeur du Musée de Physique et d'Histoire naturelle de Florence, à M. Arago. Comptes Rendus Hebdomadaires des Séances de l'Académie des Sciences 32: 635-636.

Ball, Walter William Rouse. 1893. An Essay on Newton's "Principia". London: MacMillan and Co. and New York.

Borgato, Maria Teresa. 2007. Tra teoria ed esperimenti: la deviazione dei gravi e la rotazione della terra (1789-1805). Bollettino dell'Unione Matematica Italiana. La Matematica nella Società e nella Cultura, Ser. VIII, Vol. X-A, 497-536.

Borgato, Maria Teresa. 2011. La traiettoria dei gravi nella polemica tra Borelli, Angeli e Riccioli. In Galileo e la scuola galileiana nelle Università del Seicento, edited by Luigi Pepe, 263-291. Bologna: CLUEB.

Cajori, Florian. 1901. The Unexplained Southerly Deviation of Falling Bodies. Science, New Series, Vol. 14, No. 361, 853-855.

1851. Comptes Rendus Hebdomadaires des Séances de l'Académie des Sciences, Tome trente-deuxième, Janvier-Juin. Paris: Bachelier, imprimeur-libraire, 1-1010.

Galileo, Galilei, Dialogo sopra i due Massimi Sistemi del Mondo Tolemaico, e Copernicano. Giornata Seconda, In Fiorenza: Per Gio: Batista Landini, 1632.

Galileo Galilei. 1895. Le opere di Galileo Galilei. Edizione Nazionale. Vol. V, Firenze: Tipografia di G. Barbera, 377-395.

Gauss, Johann Carl Friedrich. 1803. Gauss to Benzemberg, letter, Braunschweig, March 8, 1803. In Carl Friedrich Gauss Werke. 1867. Fünfter Band, Göttingen: Herausgegeben Von der Königlichen Gesellschaft der Wissenschaften, 497-503.

Gauss, Johann Carl Friedrich. 1804. Fundamentalgleichungen für die Bewegung Schwerer Körper aus der rotirenden Erde von Gauss. In Johann Friedrich Benzenberg. 1804. Versuche über das Gesetz des Falls, über den Widerstand der Luft und über die Umdrehung der Erde, nebst der Geschichte aller Frühren Versuche Von Galiläi bis auf 
Guglielmini. Dortmund: Bey den Gebrüdern Mallinckrodt, 363-371.

Giannini, Giulia. 2015. Gianantonio Tadini and falling bodies: a new documentary source for the reconstruction of the history of experimental proofs on the Earth's rotation. History of Science, 53 (3): 320-337.

Graney, M. Christopher. 2012. Beyond Galileo: A translation of Giovanni Battista Riccioli's experiments regarding falling bodies and "air drag", as reported in his 1651 Almagestum Novum. Preprint arXiv:1205.4663 [physics.hist-ph], 1-15.

Greenberg, John. 1987. Isaac Newton et la théorie de la Figure de la Terre. Revue d'histoire des sciences 40: 357-366.

Gregory, James. 1668. An Account of a Controversy Betwixt Stephano de Angelis, Professor of the Mathematicks in Padua, and Joh. Baptista Riccioli Jesuite as It Was Communicated Out of Their Lately Printed Books. Philosophical Transactions 3: 693698.

Guglielmini, Io. Baptistae. 1792. De Diurno Terrae Motu Experimentis Physico-Mathematicis Confirmato Opusculum. Bononiae: Ex Typographia S. Thomae Aquinatis, 1792.

Laplace, Pierre-Simon. 1803. Mémoire sur le mouvement d'un corps qui tombe d'une grande hauteur. Bulletin des Sciences, par la Société Philomathique de Paris, 75 (Prairial, an 11 de la Republique), 109-115.

Laplace, Pierre-Simon. 1805. De la chute des corps qui tombent d'une grande hauteur. Traité de Mécanique Céleste, T. IV, Chapitre V, Seconde Partie, Livre X. Paris: Chez Courcier, 294-305.

Poincaré, Henri. 1905. La Valeur de la Science. Paris: E. Flammarion éditeur.

Poincaré, Henri. 1907. The Value of Science. Authorized translation with an introduction by George Bruce Halsted, Ph. D., F.R.A.S. With a special prefatory essay. New York: The Science Press.

Reich, Ferdinand. 1832. Fallversuche über die Umdrehung der Erde angestellt auf hohe Oberbergamtliche Anordnung in dem Drei Brüderschachte bei Freiberg und herausgegeben von F. Reich, Professor der Physic an der K. S. Bergakademie. Freiberg: Verlag von J. G. Engelhardt.

Robinson Henry W. and Adams Walter. 1935. The diary of Robert Hooke 1672-1680. London: Taylor \& Francis.

Soodak, Harry and Tiersten, Martin S. 2000. Dropped objects and other motions relative to the noninertial earth. American Journal of Physics, 68 (2): 129-142.

Turnbull, Herbert Westren (ed.). 1960. The Correspondence of Isaac Newton, 1676-1687, Vol. 2. Cambridge: Cambridge University Press.

Varignon, Pierre. 1690. Nouvelles conjéctures sur la pesanteur. Paris: chez Claude Jombert. Westfall, Richard S. 1989. Newton, vol. 1, edited by A. Serafini. Torino: G. Einaudi. 